\title{
Analytical performance of the serum free light chain assay
}

\author{
Pierre-Yves Briand ${ }^{1 *}$, Olivier Decaux ${ }^{2}$, Hélène \\ Caillon ${ }^{1}$, Bernard Grosbois ${ }^{2}$, André Le Treut ${ }^{1}$ \\ and Lucienne Guenet ${ }^{1}$ \\ ${ }^{1}$ Laboratoire de Biochimie Générale et Enzymologie, CHU \\ Pontchaillou, Rennes, France \\ ${ }^{2}$ Service de Médecine Interne, CHU Hôpital Sud, Rennes, \\ France
}

\begin{abstract}
Background: The Freelite ${ }^{\mathrm{TM}}$ system for nephelometric or turbidimetric measurement of serum free light chains (FLCs) has been available since 2001. It has been valuable for the management of patients with oligosecretory myeloma, light chain myeloma and AL amyloidosis. However, there are several limitations of the method. The goal of this study was to evaluate the analytical performance of the FLC assay.

Methods: Titrated controls and clinical serum specimens were used to determine precision and post-dilution recovery. Results: As reported elsewhere, we found that the assay had several limitations, including poor post-dilution linearity and overestimation by nephelometry.

Conclusions: These data demonstrate that the results of the FLC assay must be interpreted jointly by the clinician and the biologist, taking into account the individual patient's clinical and biological characteristics.

Clin Chem Lab Med 2010;48:73-9.
\end{abstract}

Keywords: free light chains; immunoassay; monoclonal gammopathy.

\section{Introduction}

The Freelite ${ }^{\mathrm{TM}}$ assay from The Binding Site Ltd., available since 2001, can be used to determine the concentrations of $\kappa$ and $\lambda$ free light chains (FLCs). Its use is recommended by the International Myeloma Group for the diagnosis and follow-up of oligosecretory myeloma (1) and AL amyloidosis (2). The results have prognostic value at the time of diagnosis of symptomatic myeloma (3), indolent myeloma (4) and monoclonal gammapathy of undetermined significance (5). However, prospective studies are needed to determine the precise role of the FLC assay in these pathologies.

*Corresponding author: Pierre-Yves Briand, Laboratoire de Biochimie Générale et Enzymologie, 2 rue Henri Le Guilloux, 35033 Rennes Cedex 9, France

E-mail: pierreyvesbriand@yahoo.fr

Received April 4, 2009; accepted September 8, 2009;

previously published online November 25, 2009
Serum FLCs could eventually replace the need for assessment of Bence-Jones (BJ) proteinuria during the initial investigation and follow-up of monoclonal gammapathies (6). Indeed, analysis of urine has a number of drawbacks. In particular, it is highly dependent on renal glomerular and tubular function. In addition, BJ proteinuria requires a 24-h urine sample. Finally, quantification of the band observed following electrophoresis or immunofixation of urinary proteins is often difficult and imprecise (7).

The $\kappa$ and $\lambda$ FLC assay can be used on several types of automated analyzers and has been adopted for use in many laboratories worldwide. However, the results can be difficult to interpret. In particular, the lack of post-dilution linearity carries a risk of under- or overestimation. The goal of this study was to evaluate the analytical characteristics of the FLC assay, especially the influence of sample dilution, and compare our results to those reported previously.

\section{Materials and methods}

\section{Samples}

Stored serum and urine samples from patients attending Rennes University Hospital (France) for diagnosis or monitoring of monoclonal gammapathies were used for this study. Samples were obtained following routine analysis by the biochemistry laboratory with measurement of the $\kappa / \lambda$ ratio. During the study, we also used $\kappa$ and $\lambda$ quality control material provided by the manufacturer.

\section{Serum FLC analysis}

Serum FLCs were analyzed with the Dade Behring $\mathrm{BN}^{\mathrm{TM}}$ II analyzer (Siemens Healthcare Diagnostics, Marburg, Germany) using nephelometry. We used several batches of Freelite ${ }^{\mathrm{TM}}$ commercial reagents (The Binding Site Ltd., Birmingham, UK), and $\kappa$ and $\lambda$ quality control material. Serum and control specimens were analyzed according to the manufacturer's instructions $(8,9)$.

Precision of $\kappa$ and $\lambda$ FLC assay on the Dade Behring $B N^{\mathrm{Tm}} \|$ Reproducibility was estimated from results obtained with controls in successive runs. At the laboratory of biochemistry of Pontchaillou University Hospital (Rennes, France), two different concentrations of controls were analyzed at the beginning of each run and after each calibration. Between January 2007 and March 2008, measurements were performed each week and three different batches of reagents were used for both the $\kappa$ FLC and $\lambda$ FLC assay. Coefficients of variation were calculated after repeated measurements of $\kappa$ and $\lambda$ quality control material with each of the three different reagent batches.

To assess repeatability, three clinical serum samples were chosen that had low ( $\lambda$ FLC), medium ( $\kappa$ and $\lambda$ FLC) and high ( $\kappa$ FLC) concentrations of free $\kappa$ and/or $\lambda$ light chains. Coefficients of variation were calculated from the results of 20 consecutive runs. To 
verify the precision between two runs, we also chose, at random, seven other serum samples. The deviation between two runs, using the same dilution, was calculated.

Study of post-dilution recovery The dilution ranges suggested by the manufacturer overlap, indicating that, in theory, a given serum can be assayed at several different dilutions (Table 1) to obtain the same result. Using the six sample dilutions available on the $\mathrm{BN}^{\mathrm{TM}} \mathrm{II}$, the FLC working range extends from 0.41 to 20,800 $\mathrm{mg} / \mathrm{L}$ for $\lambda$ FLCs and from 0.3 to $15,200 \mathrm{mg} / \mathrm{L}$ for $\kappa$ FLCs.

To compare the results obtained for a given sample at different dilutions, 12 serum and urine samples were analyzed along with a pool of patient serum with a final $\kappa$ FLC concentration of 180 $\mathrm{mg} / \mathrm{L}$ following serial two-fold dilutions. To identify factors potentially responsible for non-agreement between results obtained at different dilutions of the same sample, controls containing known concentrations were measured using the different possible dilutions.

\section{Results}

\section{Precision of the $\kappa$ FLC and $\lambda$ FLC assay}

Reproducibility was estimated from the results obtained with controls in successive runs. The coefficients of variation for the controls ranged between $5.14 \%$ and $7.68 \%$ for $\kappa$ FLCs, and between $3.44 \%$ and $8.91 \%$ for $\lambda$ FLCs. The coefficients of variation for $\kappa$ and $\lambda$ controls are shown in Table 2 .

Reproducibality studies using three serum samples showed coefficients of variation of $6.47 \%(\lambda)$ and $6.45 \%(\kappa)$ at the

Table 1 Dilution ranges for FLC immunoassay on $\mathrm{BN}^{\mathrm{TM}} \mathrm{II}$ reported by the manufacturer (The Binding Site Ltd).

\begin{tabular}{lll}
\hline Dilution & $\begin{array}{l}\text { Dilution ranges } \\
\lambda \text { FLC }, \mathrm{mg} / \mathrm{L}\end{array}$ & $\begin{array}{l}\text { Dilution ranges } \\
\kappa \text { FLC }, \mathrm{mg} / \mathrm{L}\end{array}$ \\
\hline $1 / 1$ (urine only) & $0.08-2.60$ & $0.06-1.9$ \\
$1 / 5$ & $0.41-13.0$ & $0.3-9.5$ \\
$1 / 20$ & $1.63-52.0$ & $1.2-38.0$ \\
$1 / 100$ & $8.13-260$ & $5.9-190$ \\
$1 / 400$ & $32.5-1040$ & $23.6-760$ \\
$1 / 2000$ & $163-5200$ & $118-3800$ \\
$1 / 8000$ & $650-20,800$ & $472-15,200$ \\
\hline
\end{tabular}

standard 1/100 dilution. Coefficients of variation were $3.78 \%$ for samples with very high $\kappa$ concentrations (dilution $1 / 8000$ ), and $6.06 \%$ for low $\lambda$ concentrations (dilution $1 / 20$ ).

The precision between two runs, using seven other clinical serum samples obtained from routine samples from the laboratory, was also determined. The maximal difference between two measurements was $17.90 \%$ for $\kappa$ FLC and $10.05 \%$ for $\lambda$ FLC.

The Binding Site stipulates that control results are valid if they are within $\pm 20 \%$ of the indicated target values. All the results of repeatability and reproducibility were within the maximal accepted variation for controls, suggesting that the FLC assay using the $\mathrm{BN}^{\mathrm{TM}} \mathrm{II}$ analyzer is sufficiently reliable.

\section{Study of post-dilution recovery}

Figure 1 shows the results of the $\kappa$ FLC assay in a serial two-fold diluted pool of serum, with a theoretical starting concentration of $\kappa$ FLC of $180 \mathrm{mg} / \mathrm{L}$. The difference between the observed and theoretical concentrations ranged from $-7.8 \%$ to $+28.4 \%$. Linearity was good at the different concentrations with the $\mathrm{BN}^{\mathrm{TM}} \mathrm{II}$ analyzer. The correlation coefficients were $0.9982,0.9966,0.9979$ and 0.9996 for the $1 / 5$, $1 / 20,1 / 100$ and $1 / 400$ dilutions, respectively. It can also be noted that for each theoretical concentration, the observed concentration increased with the dilution factor. Also, difference between the observed and theoretical concentrations exceeded $20 \%$ for two of eight samples.

To further study post-dilution recovery, 12 clinical serum and urine samples were also analyzed. Based on the manufacturer's overlapping working ranges, two or three dilutions were possible for some samples and the results are shown in Table 3.

Large differences were observed with low concentrations of FLC (e.g., dilution 1/5, 1/20, 1/100: patients 3 and 12 for $\lambda$ FLC). In contrast, for high concentrations of FLC (e.g., dilutions 1/400, 1/2000 and 1/8000: patient 4 for $\lambda$ FLC, patient 1 for $\kappa$ FLC) the differences were less important. Few such differences were seen with urine samples (patients 8 and 10). In addition, the observed differences between dilutions of samples of similar concentrations were highly variable from patient to patient (e.g., patients 1 and 9 for $\lambda$ FLC)

Table 2 Estimation of reproducibility.

\begin{tabular}{|c|c|c|c|c|c|c|c|c|c|c|c|c|}
\hline & \multicolumn{4}{|c|}{ 1st control batch } & \multicolumn{4}{|c|}{ 2nd control batch } & \multicolumn{4}{|c|}{ 3rd control batch } \\
\hline & \multicolumn{2}{|c|}{$\begin{array}{l}\kappa(21 / 01 / 07- \\
06 / 04 / 07)\end{array}$} & \multicolumn{2}{|c|}{$\begin{array}{l}\lambda(12 / 01 / 07- \\
30 / 03 / 07)\end{array}$} & \multicolumn{2}{|c|}{$\begin{array}{l}\kappa(13 / 04 / 07- \\
02 / 11 / 07)\end{array}$} & \multicolumn{2}{|c|}{$\begin{array}{l}\lambda(16 / 04 / 07- \\
19 / 10 / 07)\end{array}$} & \multicolumn{2}{|c|}{$\begin{array}{l}\kappa(09 / 11 / 07- \\
28 / 03 / 08)\end{array}$} & \multicolumn{2}{|c|}{$\begin{array}{l}\lambda(26 / 10 / 07- \\
28 / 03 / 08)\end{array}$} \\
\hline & Level 1 & Level 2 & Level 1 & Level 2 & Level 1 & Level 2 & Level 1 & Level 2 & Level 1 & Level 2 & Level 1 & Level 2 \\
\hline Mean, mg/L & 16.28 & 31.72 & 26.11 & 58.32 & 16.41 & 28.71 & 28.75 & 56.89 & 14.14 & 25.54 & 27.53 & 56.84 \\
\hline SD & 1.25 & 1.63 & & 2.00 & 1.26 & 1.52 & 2.56 & 3.23 & 1.08 & 1.73 & 2.34 & 2.42 \\
\hline $\begin{array}{l}\text { Coefficient of } \\
\text { variation, } \%\end{array}$ & 7.68 & 5.14 & 4.85 & 3.44 & 7.67 & 5.31 & 8.91 & 5.67 & 7.67 & 6.76 & 8.49 & 4.25 \\
\hline Target, mg/L & 16.20 & 31.00 & 27.20 & 59.80 & 16.50 & 29.80 & 29.90 & 56.90 & 13.60 & 27.50 & 27.70 & 55.60 \\
\hline
\end{tabular}

Two different concentrations of control were analyzed at the beginning of each run and after each calibration. Between January 2007 and March 2008, runs were performed each week and three different batches of controls were used for both $\kappa$ and $\lambda$ FLC assays. 


\section{A}

\begin{tabular}{|c|c|c|c|c|c|c|c|c|c|}
\hline $\begin{array}{r}\text { Theoretical } \\
\text { concentration, } \\
\kappa \mathrm{FLC}, \mathrm{mg} / \mathrm{L}\end{array}$ & 180 & 90 & 45 & 22.5 & 12.25 & 6.125 & 3.0625 & 1.53125 & 0.765625 \\
\hline $1 / 2000$ & $\begin{array}{c}211 \\
+17.2 \%\end{array}$ & 1 & 1 & 1 & 1 & 1 & I & I & I \\
\hline $1 / 400$ & $\begin{array}{l}\mathbf{1 8 0} \\
0 \%\end{array}$ & $\begin{array}{r}94.3 \\
+4.8 \%\end{array}$ & $\begin{array}{r}\mathbf{5 3 . 4} \\
+18.7 \%\end{array}$ & $\begin{array}{r}\mathbf{2 8 . 9} \\
+28.4 \%\end{array}$ & 1 & 1 & 1 & 1 & 1 \\
\hline $1 / 100$ & I & $\begin{array}{r}90 \\
0 \%\end{array}$ & $\begin{array}{r}49.5 \\
+10 \%\end{array}$ & $\begin{array}{c}25.2 \\
+12 \%\end{array}$ & $\begin{array}{c}14.4 \\
+17.6 \%\end{array}$ & $\begin{array}{r}7.55 \\
+23.3 \%\end{array}$ & 1 & 1 & I \\
\hline $1 / 20$ & 1 & I & 1 & $\begin{array}{c}\mathbf{2 2 . 7} \\
+0.9 \%\end{array}$ & $\begin{array}{r}11.3 \\
-7.8 \%\end{array}$ & $\begin{array}{c}6.2 \\
+1.2 \%\end{array}$ & $\begin{array}{c}3.32 \\
+8.4 \%\end{array}$ & $\begin{array}{r}1.57 \\
+2.5 \%\end{array}$ & 1 \\
\hline $1 / 5$ & 1 & 1 & 1 & 1 & 1 & $\begin{array}{r}5.69 \\
-7.1 \%\end{array}$ & $\begin{array}{c}\mathbf{2 . 7 5} \\
-11.4 \%\end{array}$ & $\begin{array}{r}1.57 \\
+2.5 \%\end{array}$ & $\begin{array}{c}\mathbf{0 . 8 1} \\
+5.7 \%\end{array}$ \\
\hline
\end{tabular}

B

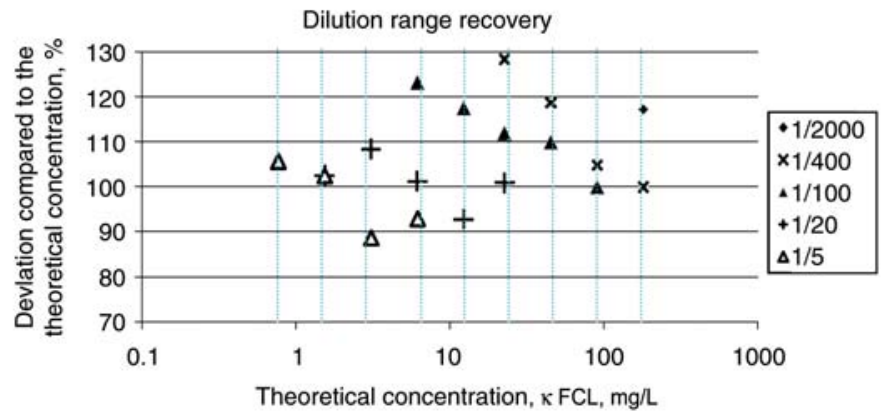

Figure 1 Difference between the observed and theoretical concentrations in a serial two-fold diluted pool of serum (theoretical starting concentration of $\kappa \mathrm{FLC}=180 \mathrm{mg} / \mathrm{L}$ ).

(A) Observed concentration according to the theoretical concentration of $\kappa$ FLC and working dilutions. The percentages correspond to the calculated deviation compared to the theoretical concentration. (B) Deviation compared to the theoretical concentration and working dilution.

and difficult to predict. This issue could pose a problem for interpreting results for some patients, as these discrepancies could lead to an erroneous conclusion that the $\kappa / \lambda$ ratio is abnormal, when in fact the ratio is normal.

To identify potential factors responsible for the discrepancies observed with the same sample assayed at different dilutions, controls containing known concentrations were assayed at the different possible dilutions. The results are shown in Table 4. The observed differences, ranging from $-9.93 \%$ to $+2.58 \%$, were far smaller with the protein-free controls and with the protein-poor urine samples compared with serum. The problems observed with serum are probably due to interference from matrix effects at low dilutions, possibly owing to the high protein content of serum. However, other factors may be responsible for the discrepant results observed at different dilutions.

\section{Risk of overestimation}

During our study, we observed the case of a patient monitored for myeloma with a $\kappa$ FLC component. Electrophoresis and serum protein immunofixation revealed the presence of two monoclonal $\lambda$ FLCs migrating with the gammaglobulins (data not shown). During follow-up, the FLC assay overestimated concentrations on two occasions (respectively 19,900 and $12,700 \mathrm{mg} / \mathrm{L}$ by nephelemetry, 5000 and $7300 \mathrm{mg} / \mathrm{L}$ by electrophoresis). Values obtained with the Freelite ${ }^{\mathrm{TM}}$ method in these patients did not correspond with the actual concentrations obtained by integration of the electrophoretic profile.

\section{Discussion}

\section{Analytical performance of FLC assay}

The FLC assay can detect a monoclonal component in certain situations where previously it was impossible. Thus, this test makes an important contribution to patient management in such cases. However, the FLC assay has several limitations.

We identified a number of practical problems, confirming data published elsewhere. Many of these issues are common to all new immunoassays, but they are aggravated by the fact that there is only one commercial reagent and no internatio- 
Table 3 FLC concentrations at different dilutions on the $\mathrm{BN}^{\mathrm{TM}} \mathrm{II}$ for serum and urine samples.

\begin{tabular}{|c|c|c|c|c|c|c|}
\hline \multirow[t]{2}{*}{ Patients } & \multicolumn{3}{|l|}{ FLC $\lambda$} & \multicolumn{3}{|l|}{ FLC $\kappa$} \\
\hline & Dilution & $\begin{array}{l}\text { Concentration, } \\
\mathrm{mg} / \mathrm{L}\end{array}$ & $\begin{array}{l}\text { Recovery, } \\
\%\end{array}$ & Dilution & $\begin{array}{l}\text { Concentration, } \\
\mathrm{mg} / \mathrm{L}\end{array}$ & $\begin{array}{l}\text { Recovery, } \\
\%\end{array}$ \\
\hline \multirow[t]{3}{*}{1 Serum } & 100 & 9.77 & 197.4 & 2000 & 213 & 130.7 \\
\hline & 20 & 9.18 & 185.5 & 400 & 200 & 122.7 \\
\hline & 5 & 4.95 & 100 & 100 & 163 & 100 \\
\hline \multirow[t]{2}{*}{2 Serum } & 100 & 13.3 & 139 & 100 & 11.6 & 210.9 \\
\hline & 20 & 9.57 & 100 & 20 & 5.5 & 100 \\
\hline \multirow[t]{3}{*}{3 Serum } & 100 & 9.5 & 372 & 100 & 9.5 & 224.60 \\
\hline & 20 & 5.9 & 232.3 & 20 & 4.23 & 100 \\
\hline & 5 & 2.54 & 100 & & & \\
\hline \multirow[t]{2}{*}{4 Serum } & 40,000 & 13,300 & 74.3 & 100 & 18.3 & 205.2 \\
\hline & 8000 & 17,900 & 100 & 20 & 8.92 & 100 \\
\hline \multirow[t]{3}{*}{5 Serum } & 100 & 11.9 & 255.9 & 100 & 15.5 & 118.3 \\
\hline & 20 & 7.28 & 156.6 & 20 & 13.1 & 100 \\
\hline & 5 & 4.65 & 100 & & & \\
\hline \multirow[t]{3}{*}{6 Serum } & 400 & 45.2 & 168.7 & 100 & 11.3 & 200.7 \\
\hline & 100 & 44.2 & 164.9 & 20 & 5.63 & 100 \\
\hline & 20 & 26.8 & 100 & & & \\
\hline \multirow[t]{3}{*}{7 Serum } & 100 & 10.5 & 160.6 & 100 & 15.4 & 181.2 \\
\hline & 20 & 14.2 & 217.1 & 20 & 8.5 & 100 \\
\hline & 5 & 6.54 & 100 & & & \\
\hline \multirow[t]{2}{*}{8 Urine } & 8000 & 2090 & 78.3 & 100 & 16.3 & 87.2 \\
\hline & 2000 & 2670 & 100 & 20 & 18.7 & 100 \\
\hline \multirow{3}{*}{9 Serum } & 100 & 17.8 & 327.8 & 100 & 50.8 & 196.1 \\
\hline & 20 & 11 & 202.6 & 20 & 25.9 & 100 \\
\hline & 5 & 5.43 & 100 & & & \\
\hline \multirow[t]{2}{*}{10 Urine } & 100 & 8.42 & 73.9 & 400 & 66.4 & 138 \\
\hline & 20 & 11.4 & 100 & 100 & 48.1 & 100 \\
\hline \multirow[t]{2}{*}{11 Serum } & 100 & 16.8 & 158.5 & 100 & 15 & 175.4 \\
\hline & 20 & 10.6 & 100 & 20 & 8.55 & 100 \\
\hline \multirow[t]{3}{*}{12 Serum } & 100 & 10.1 & 381.1 & 100 & 13.5 & 230.4 \\
\hline & 20 & 6.44 & 243 & 20 & 5.86 & 100 \\
\hline & 5 & 2.65 & 100 & & & \\
\hline
\end{tabular}

The results obtained at the lowest dilution serve as basis (100\%) for the calculation of percent recovery.

Table 4 FLC concentrations for $\kappa$ and $\lambda$ controls, analyzed at several dilutions.

\begin{tabular}{llrll}
\hline Controls & $\begin{array}{l}\text { Target, } \\
\mathrm{mg} / \mathrm{L}\end{array}$ & Dilution & $\begin{array}{l}\text { Concentration, } \\
\mathrm{mg} / \mathrm{L}\end{array}$ & $\begin{array}{l}\text { Deviation, } \\
\%\end{array}$ \\
\hline$\lambda$ low & 27.2 & 100 & 24.5 & -9.93 \\
& & 20 & 25.8 & -5.15 \\
$\lambda$ high & 59.8 & 100 & 58.6 & -2.01 \\
& & 400 & 57.7 & -3.51 \\
$\kappa$ low & \multirow{2}{*}{16.2} & 100 & 15.3 & -5.56 \\
& & 20 & 16.3 & 0.62 \\
$\kappa$ high & \multirow{2}{*}{31} & 100 & 29.4 & -5.16 \\
& & 400 & 31.8 & 2.58 \\
\hline
\end{tabular}

nal standard. Clinicians and biologists must be aware of these limitations if they are to avoid misinterpreting results.

Effects of serum dilution on the FLC assay results Analysis of several serum samples on the $\mathrm{BN}^{\mathrm{TM}} \mathrm{II}$ device showed poor post-dilution linearity, with the observed values increasing consistently with the dilution factor. The discrepancies observed for a given sample measured at several dilutions were highly variable from patient to patient, leading to the risk of over- or underestimation of FLC concentrations. Indeed, the dilution chosen for a given sample (based on the patient's historical values) is not always optimal, and the working ranges provided by the manufacturer for different dilutions overlap. This means that it is not always necessary to re-run the sample at a higher or lower dilution. To minimize the risk of error, laboratories using the Freelite ${ }^{\mathrm{TM}}$ method should use non-overlapping working ranges for each dilution. Table 5 shows the non-overlapping working ranges used in our laboratory. Another way to limit the risk of error would be to use the starting dilution recommended by the manufacturer, i.e., 1/100, without taking into account the patient's historical values. Unfortunately, this is a costly option.

Similar conflicts between different dilutions of a given sample were also reported in 2007 with the Immage $^{\mathrm{TM}}$ Protein System (Beckman Coulter Inc., Fullerton, CA, USA) (10). 
Table 5 Non-overlapping working ranges used in the laboratory of biochemistry of Pontchaillou hospital (Rennes, France) for the FLC immunoassay.

\begin{tabular}{lll}
\hline Dilution & $\begin{array}{l}\text { Dilution range } \\
\lambda, \mathrm{mg} / \mathrm{L}\end{array}$ & $\begin{array}{l}\text { Dilution range } \\
\kappa, \mathrm{mg} / \mathrm{L}\end{array}$ \\
\hline $1 / 5$ & $0.3-1.2$ & $0.4-1.6$ \\
$1 / 20$ & $1.2-5.9$ & $1.6-8.1$ \\
$1 / 100$ & $5.9-190$ & $8.1-260$ \\
$1 / 400$ & $190-760$ & $260-1040$ \\
$1 / 2000$ & $760-3800$ & $1040-5200$ \\
$1 / 8000$ & $3800-15,200$ & $5200-20,800$ \\
\hline
\end{tabular}

In this study, the analysis of several dilutions of serum from 16 patients with monoclonal or polyclonal FLCs showed a rise in the observed values as the dilution factor increased, again creating a risk of over- or underestimation. Several factors may explain these discrepancies between different dilutions, as shown by our comparison of patient serum and urine samples and titrated control samples. Our results show a matrix effect with serum, the high protein concentration in the reaction mixture possibly interfering with the antigen/ antibody reaction at low dilutions.

In their 2007 study, Tate et al. focused not on the influence of the reaction medium, but instead on the nature of the FLC participating in the immunochemical reaction. Comparison of the responses obtained with samples containing polyclonal FLC and samples containing monoclonal FLC showed that the results varied when the samples were diluted. Post-dilution linearity was not as good for monoclonal FLC compared with FLC, the latter behaving more like the calibrator which is composed of polyclonal FLC. This might occur because monoclonal FLC can recognize only one antibody species among the polyclonal antibodies constituting the assay reagent, leading to antigen-to-antibody excess. Thus, for a given amount of polyclonal reagent, antigen excess occurs at lower concentrations with monoclonal FLCs than with polyclonal FLCs. In addition, the differences were more marked with $\kappa$ FLC compared with $\lambda$ FLC (10). Another study, published in 1991, described problems of post-dilution linearity with a monoclonal FLC assay (11).

Risk of overestimation In our study, we observed in a myeloma patient a case of overestimation of the FLC concentration measured with nephelometry compared to results obtained by integration of the electrophoretic profile. Polymerization of intact immunoglobulins (Igs), especially IgM and $\operatorname{IgA}$, is a frequent phenomenon and hinders their quantification. Similarly, polymerization of FLCs can sometimes lead to overestimation, as described in the literature (12-15). It has been shown that FLCs can exist in multimeric forms, in addition to their classical presentation as $\kappa$ FLC monomers and $\lambda$ FLC dimers $(14,16)$. Moreover, complex formation has been reported between $\alpha_{1}$-antitrypsin and $\kappa$ FLC, leading to overestimation (17). In 2002, Abraham et al. described a case of monoclonal $\lambda$ FLC overestimation by nephelometry, owing to the presence of trimolecular aggregates composed of three $\lambda$ FLC dimers (14). Likewise, urine assays often give values far above the urine total protein concentration (12).

Influence of the reagent batch on FLC assay results Another study published by Tate in 2007 described significant variability according to the reagent batch (10). This means that the results obtained for a given patient monitored for several years will be difficult to interpret, as several different reagent batches will inevitably be used. Clinicians must be aware that variations in the $\kappa / \lambda$ ratio can occur independent of any spontaneous or treatment-induced changes in disease. In addition, in a laboratory like ours where $\sim 30$ FLC assays are performed each week to primarily monitor patients with hematological disorders, ideally a frozen aliquot of each patient's previous serum sample should be retested at the same time as the latest sample in order to compensate for inter-batch variability, but this approach is difficult due to issues of cost and storage and storage of samples.

A case of "non-reaction", with several reagent batches of the $\kappa$ FLC assay was noted in the study by Tate et al. involving a 70-year-old patient with $\kappa$ FLC multiple myeloma. The reason why the monoclonal $\kappa$ FLC did not react with several reagent batches was not identified. This first reported case of "non-reaction", in a patient with a monoclonal $\kappa$ FLC clearly shows that polyclonal reagents may not recognize all $\kappa$ or $\lambda$ FLCs (whether monoclonal or polyclonal), owing to their marked structural diversity (10).

Influence of the choice of analyzer on the results of FLC assay Pairwise comparisons of automated analyzers for FLC assay have been published. In 2003, Tate et al. (12) compared the $\mathrm{BN}^{\mathrm{TM}}$ ProSpec (Siemens Healthcare Diagnostics, Marburg, Germany) with the Immage ${ }^{\mathrm{TM}}$ Protein System. The correlation study used serum from 37 patients. With low values of $\kappa$ FLC and $\lambda$ FLC (3.5-19 $\mathrm{mg} / \mathrm{L}$ and 3.7-26 $\mathrm{mg} / \mathrm{L}$, respectively), the corresponding correlation coefficients were 0.81 and 0.87 . With higher values of $\kappa$ and $\lambda$ FLCs $(20-168 \mathrm{mg} / \mathrm{L}$ and $29-100 \mathrm{mg} / \mathrm{L}$, respectively), the corresponding correlation coefficients were better $(0.95$ and 0.96). In a study published by Pattenden et al. (18) comparing serum FLC values obtained with the Dade Behring $\mathrm{BN}^{\mathrm{TM}} \mathrm{II}$ and the Olympus AU400 ${ }^{\circledR}$ (Olympus Diagnostic Systems, Hamburg, Germany) from 112 patients, higher values were obtained with the $\mathrm{BN}^{\mathrm{TM}} \mathrm{II}$ device than with the $\mathrm{AU} 400^{\circledR}$, with the difference being more marked for $\lambda$ FLCs. Thus, although the recommended reference ranges are the same for all analyzers (18), the results of Tate and Pattenden show that reference ranges must be established specifically for each device.

The divergent results obtained with different analyzers may be due in part to the use of nephelometry with some devices $\left(\mathrm{BN}^{\mathrm{TM}} \mathrm{II}, \mathrm{BN}^{\mathrm{TM}}\right.$ ProSpec, and Immage $\left.{ }^{\mathrm{TM}}\right)$ and turbidimetry on others (Olympus AU400 ${ }^{\circledR}$ ). However, Tate et al. also reported that two analyzers using the same detection 
method (nephelometry) could give different results, showing that other factors contribute to this variability (12).

Risk of cross-reactions with intact Ig If the sample contains intact Igs, with more bound light chains compared with FLC, the reliability of the results will depend on the specificity of the antiserum. The non-specificity according to the manufacturer is $<0.4 \%$, which, as pointed out by Nakano et al., raises uncertainties, particularly for FLC к (19).

\section{Practical implications for routine FLC assay}

This study of the analytical performance of FLC assay confirms certain limitations of this method. Laboratory staff and clinicians must be aware of the risk of over- or underestimation, depending on the sample dilution and the use of different reagent batches or analyzers, as well as of the risk of reaction failure with some monoclonal FLCs. Indeed, this variability can lead to major errors in measurement of $\kappa$ or $\lambda$ FLC concentrations and the ratio. If these limitations are not taken into account, they can lead to an incorrect diagnosis or misjudgment of the response to treatment. FLCs, being the only marker available to monitor changes in oligosecretory and non-secretory multiple myeloma and AL amyloidosis, must have results that are as reproducible as possible.

To minimize the risk of error, we recommend the following:

- Non-overlapping working ranges should be used for each dilution, thus reducing the imprecision due to poor postdilution linearity (Table 5). A sample must be systematically re-run at a lower or higher dilution if the result does not fall between these predefined limits.

- Ideally, local reference ranges should be established in each laboratory using the Freelite ${ }^{\mathrm{TM}}$ technique. Indeed, several studies have shown that significantly different results may be obtained with different analyzers.

- For a given patient, all $\kappa$ and $\lambda$ FLC measurements should ideally be performed in the same laboratory with the same analyzer, leaving only the problem of inter-batch variability.

- It is recommended that variations in serum FLC concentration of $<50 \%$ should not be considered as proof of disease progression or of a response to treatment in patients with $\mathrm{AL}$ amyloidsis (20) or multiple myeloma (4).

- Dialogue between the biologist and clinician is essential to ensure optimal assay conditions and interpretation. The biologist must be provided with clinical information at the time the sample is received, as knowledge of the disease and ongoing treatments will help to select the best assay dilutions. This information will help limit the number of reruns and yield the most precise result possible. For example, a higher dilution will be used initially if the patient has signs of myeloma relapse. In turn, the biologist must inform the clinician of the analytical limits of the assay in order to ensure that the results are interpreted correctly, in conjunction with the patient's clinical characteristics. Given the high variability observed with low dilutions, clinicians should be advised to ignore variations in the $\kappa / \lambda$ ratio in patients with below normal FLC concentrations.

\section{Conflict of interest statement}

Authors' conflict of interest disclosure: The authors stated that there are no conflicts of interest regarding the publication of this article.

Research funding: None declared.

Employment or leadership: None declared.

Honorarium: None declared.

\section{References}

1. Durie BG, Harousseau JL, Miguel JS, Bladé J, Barlogie B, Anderson K, et al. International uniform response criteria for multiple myeloma. Leukemia 2006;20:1467-73.

2. Guidelines Working Group of UK Myeloma Forum. Guidelines on the diagnosis and management of $\mathrm{AL}$ amyloidosis. $\mathrm{Br} \mathrm{J}$ Haematol 2004;125:681-700.

3. Mead GP, Carr-Smith HD, Drayson MT, Morgan GJ, Child JA, Bradwell AR. Serum free light chains for monitoring multiple myeloma. Br J Haematol 2004;126:348-54.

4. Dispenzieri A, Zhang L, Katzmann JA, Snyder M, Blood E, DeGoey R, et al. Appraisal of immmunoglobulin free light chain as a marker of response. Blood 2008;111:4908-15.

5. Rajkumar SV, Kyle RA, Therneau TM, Melton III LJ, Bradwell AR, Clark RJ, et al. Serum free light chain ratio is an independent risk factor for progression in monoclonal gammopathy of undetermined significance. Blood 2005;106:812-7.

6. Katzmann JA, Dispenzieri A, Kyle R, Snyder MR, Plevak BS, Larson DR, et al. Elimination of the need for urine studies in the screening algorithm for monoclonal gammopathies by using serum immunofixation and free light chain assays. Mayo Clin Proc 2006;81:1575-8.

7. Guenet L, Decaux O, Lechartier H, Ropert M, Grosbois B. Intérêt du dosage des chaînes légères libres d'immunoglobulines sériques pour le diagnostic et le suivi des gammapathies monoclonales. Rev Med Int 2007;28:689-97.

8. Freelite human kappa free kit for use on the Dade Behring Nephelometer (BN $\left.{ }^{\mathrm{TM}} \mathrm{II}\right)$. LK016.T. The Binding Site Ltd., 2008.

9. Freelite human lambda free kit for use on the Dade Behring Nephelometer (BN $\left.{ }^{\mathrm{TM}} \mathrm{II}\right)$. LK018.T. The Binding Site Ltd., 2008.

10. Tate J, Mollee P, Dimeski G, Carter A, Gill D. Analytical performance of serum free light-chain assay during monitoring of patients with monoclonal light-chain diseases. Clin Chim Acta 2007;376:30-6.

11. Tillyer CR, Iqbal J, Raymond J, Gore M, McIlwain TJ. Immunoturbidimetric assay for estimating free light chains of immunoglobulins in urine and serum. J Clin Pathol 1991;44:466-71.

12. Tate J, Gill D, Cobcroft R, Hickman PE. Practical considerations for the measurement of free light chains in serum. Clin Chem 2003;49:1252-7.

13. Bradwell AR, Carr-Smith HD, Mead GP, Tang LX, Showell PJ, Drayson MT, et al. Highly sensitive, automated immunoassay 
for immunoglobulin free light chains in serum and urine. Clin Chem 2001;47:673-80.

14. Abraham RS, Charlesworth MC, Owen BA, Benson LM, Katzmann JA, Reeder CB. Trimolecular complexes of $\lambda$ light chains dimers in serum of a patient with multiple myeloma. Clin Chem 2002;48:1805-11.

15. Lopez J, Dauwalder O, Joly P, Dimet I, Bienvenu J, Bernon H. Intérêts et limites des dosages sériques et urinaires des chaînes légères libres pour le diagnostic et le suivi des dysglobulinémies monoclonales. Ann Biol Clin 2006;64:287-97.

16. Mead GP, Stubbs PS, Carr-Smith HD, Drew RL, Bradwell AR. Nephelometric measurement of serum free light chains in nonsecretory myeloma [abstract]. Clin Chem 2002;48(Suppl 6):A23.

17. Chapuis-Cellier C, Chazaud A, Foray V, Troncy J. Apparent discrepencies in the quantification of free light chains in serum of patients presenting with a monoclonal gammmopathy. Haematologica 2005;90:109.

18. Pattenden RJ, Rogers SY, Wenham PR. Serum free light chains; the need to establish local reference intervals. Ann Clin Biochem 2007;44:512-5.

19. Nakano T, Miyazaki S, Takahashi H, Matsumori A, Maruyama $\mathrm{T}$, Komoda $\mathrm{T}$, et al. Immunochemical quantification of free immunoglobulin light chains from an analytical perspective. Clin Chem Lab Med 2006;44:522-32.

20. Gertz MA, Comenzo R, Falk RH, Fermand JP, Hazenberg BP, Hawkins PN, et al. Definition of organ involvement and treatment response in immunoglobulin light chain amyloidosis (AL): a consensus opinion from the 10th International Symposium on Amyloid and Amyloidosis. Am J Hematol 2005; 79:319-28. 